\title{
Bosonic supersymmetry? Getting fooled at the CERN LHC
}

\author{
Hsin-Chia Cheng \\ Enrico Fermi Institute, The University of Chicago, Chicago, Illinois 60637 \\ Konstantin T. Matchev \\ Department of Physics, University of Florida, Gainesville, Florida 32611 \\ and TH Division, CERN, Geneva 23, CH-1211, Switzerland \\ Martin Schmaltz \\ Department of Physics, Boston University, Boston, Massachusetts 02215
}

(Received 3 June 2002; published 23 September 2002)

\begin{abstract}
We define a minimal model with universal extra dimensions, and begin to study its phenomenology. The collider signals of the first Kaluza-Klein (KK) level are surprisingly similar to those of a supersymmetric model with a nearly degenerate superpartner spectrum. The lightest KK particle (LKP) is neutral and stable because of KK parity. KK excitations cascade decay to the LKP yielding missing energy signatures with relatively soft jets and leptons. Level $2 \mathrm{KK}$ modes may also be probed via their KK number violating decays to standard model particles. In either case we provide initial estimates for the discovery potential of the Fermilab Tevatron and the CERN Large Hadron Collider.
\end{abstract}

DOI: 10.1103/PhysRevD.66.056006

PACS number(s): 11.10.Kk, 14.80.-j, 04.50.+h

\section{INTRODUCTION}

The new ideas of extra dimensions and localized gravity have recently attracted a lot of interest. They not only offer exciting new avenues for theoretical exploration but also predict signals which can soon be tested at the upcoming collider experiments at the Fermilab Tevatron and the CERN Large Hadron Collider (LHC).

The focus of this paper is on universal extra dimensions (UEDs) [1], a model in which all standard model fields propagate in extra dimensions of size $R^{-1} \sim \mathrm{TeV}$. Although there are many theoretical reasons for studying UEDs (electroweak symmetry breaking [2], proton decay [3], the number of generations [4], neutrino masses [5], etc.), we are primarily motivated by their collider phenomenology. Experimental bounds allow Kaluza-Klein (KK) modes in UEDs to be as light as a few hundred $\mathrm{GeV}[1,6,7]$. The production cross section at the LHC for KK excitations of quarks and gluons weighing only a few hundred $\mathrm{GeV}$ is enormous. However, as we discuss in this paper, their subsequent detection is nontrivial because they decay nearly invisibly. The phenomenology of UEDs shows interesting parallels to supersymmetry. Every standard model field has KK partners. The lowest level KK partners carry a conserved quantum number, KK parity, which guarantees that the lightest KK particle (LKP) is stable. Heavier KK modes cascade decay to the LKP by emitting soft standard model particles. The LKP escapes detection, resulting in missing energy signals.

In the following section we define minimal universal extra dimensions (MUEDs). The model is defined in five dimensions with one dimension compactified on an $S_{1} / Z_{2}$ orbifold. All fields propagate in the bulk and have KK modes with masses approximately equal to the compactification scale. The Lagrangian of the model includes interactions which are localized at the boundaries of the orbifold. These boundary terms lead to mass splittings between KK modes and affect their decays. In Secs. III and IV we discuss the phenomenology of the first and second level KK states, respectively. We identify possible decay modes and branching ratios, and we estimate the discovery reach at the Tevatron and the LHC. Section V contains our conclusions and speculations about the cosmology of UEDs.

\section{MINIMAL UNIVERSAL EXTRA DIMENSIONS}

The simplest UED scenario has all of the standard model fields (no supersymmetry) propagating in a single extra dimension. In $4+1$ dimensions, the fermions $\left[Q_{i}, u_{i}, d_{i}, L_{i}, e_{i}, i=1,2,3\right.$, where upper (lower) case letters represent $S U(2)$ doublets (singlets)] are four-component and contain both chiralities when reduced to $3+1$ dimensions. To produce a chiral 4D spectrum, we compactify the extra dimension on an $S_{1} / Z_{2}$ orbifold. Fields which are odd under the $Z_{2}$ orbifold symmetry do not have zero modes, hence the unwanted fields (zero modes of fermions with the wrong chiralities and the 5th component of the gauge fields) can be projected out. The remaining zero modes are just the standard model particles in $3+1$ dimensions.

The full Lagrangian of the theory comprises both bulk and boundary interactions. Gauge and Yukawa couplings and the Higgs potential are contained in the bulk Lagrangian in one-to-one correspondence with the couplings of the standard model. The boundary Lagrangian interactions are localized at the orbifold fixed points and do not respect five dimensional Lorentz invariance.

Ignoring the localized terms for the moment, the mass of the $n$th $\mathrm{KK}$ mode is

$$
m_{n}^{2}=\frac{n^{2}}{R^{2}}+m_{0}^{2},
$$


where $R$ is the radius of the compact dimension, and $m_{0}$ is the zero mode mass. The spectrum at each KK level is highly degenerate except for particles with large zero mode masses $(t, W, Z, h)$. The bulk interactions preserve the 5th dimensional momentum (KK number). The corresponding coupling constants among $\mathrm{KK}$ modes are simply equal to the SM couplings (up to normalization factors such as $\sqrt{2}$ ). The Feynman rules for the KK modes can easily be derived (e.g., see Refs. $[8,9])$.

In contrast, the coefficients of the boundary terms are not fixed by standard model couplings and correspond to new free parameters. In fact, they are renormalized by the bulk interactions and hence are scale dependent [10,11]. One might worry that this implies that all predictive power is lost. However, since the wave functions of standard model fields and KK modes are spread out over the extra dimension and the new couplings only exist on the boundaries, their effects are volume suppressed. We can get an estimate for the size of these volume suppressed corrections with naive dimensional analysis by assuming strong coupling at the cutoff. The result is that the mass shifts to KK modes from boundary terms are numerically equal to corrections from loops $\delta m_{n}^{2} / m_{n}^{2}$ $\sim g^{2} / 16 \pi^{2}$.

We will assume that the boundary terms are symmetric under the exchange of the two orbifold fixed points, which preserves the KK parity discussed below. Most relevant to the phenomenology are localized kinetic terms for the SM fields, such as

$$
\frac{\delta\left(x_{5}\right)+\delta\left(x_{5}-\pi R\right)}{\Lambda}\left[G_{4}\left(F_{\mu \nu}\right)^{2}+F_{4} \bar{\Psi} i D \Psi+F_{5} \bar{\Psi} \gamma_{5} \partial_{5} \Psi\right],
$$

where the dimensionless coefficients $G_{4}$ and $F_{i}$ are arbitrary and not universal for the different standard model fields. These terms are important phenomenologically for several reasons: (i) they split the near-degeneracy of KK modes at each level, (ii) they break KK number conservation down to a KK parity under which modes with odd KK numbers are charged, (iii) they introduce possible new flavor violation.

Since collider signatures depend strongly on the values of the boundary couplings it is necessary to be definite and specify them. A reasonable Ansatz is to take flavor-universal boundary terms. Nonuniversalities would give rise to flavor changing neutral currents as in supersymmetry with flavor violating scalar masses. This still leaves a large number of free parameters. For definiteness, and also because we find the resulting phenomenology especially interesting, we make the assumption that all boundary terms are negligible at some scale $\Lambda>R^{-1}$. This defines our model.

Note that this is completely analogous to the case of the minimal supersymmetric standard model (MSSM) where one has to choose a set of soft supersymmetry breaking couplings at some high scale, before studying the phenomenology. Different Ansätze for the parameters can be justified by different theoretical prejudices but ultimately one should use experimental data to constrain them. In a sense, our choice of boundary couplings may be viewed as analogous to the simplest minimal supergravity boundary condition-universal

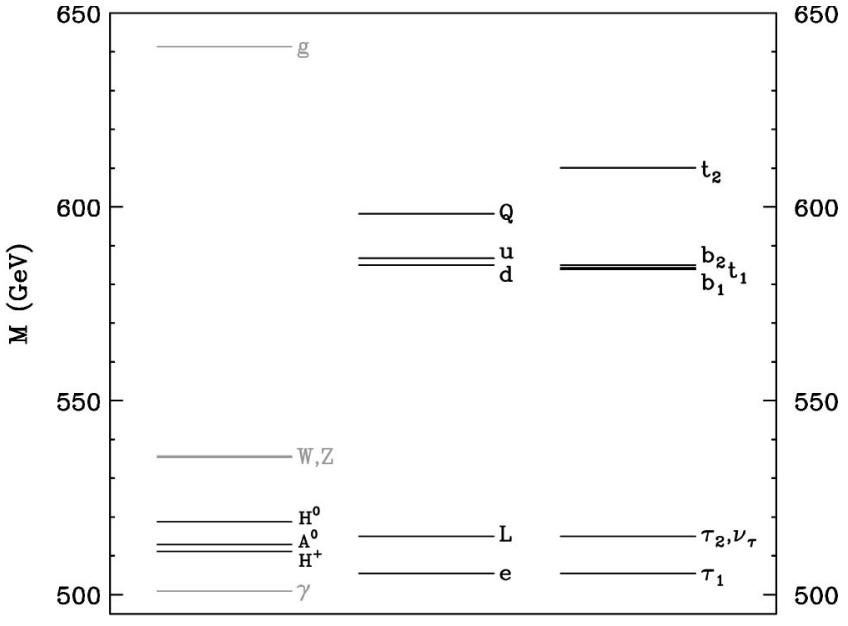

FIG. 1. One-loop corrected mass spectrum of the first KK level in MUEDs for $R^{-1}=500 \mathrm{GeV}, \Lambda R=20$ and $m_{h}=120 \mathrm{GeV}$.

scalar and gaugino masses. Thus the model of MUEDs is extremely predictive and has only three free parameters:

$$
\left\{R, \Lambda, m_{h}\right\},
$$

where $m_{h}$ is the mass of the standard model Higgs boson.

The low energy KK spectrum of MUEDs depends on the boundary terms at low scales which are determined from the high energy parameters through the renormalization group. Since the corrections are small we use the one-loop leading $\log$ approximations. In addition to the boundary terms we also take into account the nonlocal radiative corrections to KK masses. All these were computed at one-loop in [10].

A typical spectrum for the first level KK modes is shown in Fig. 1. Figure 2 shows the dependence of the splittings between first level KK modes on the cutoff scale $\Lambda$. Typically, the corrections for KK modes with strong interactions are $>10 \%$ while those for states with only electroweak interactions are a few percent. We find that the corrections to the masses are such that $m_{g_{n}}>m_{Q_{n}}>m_{q_{n}}>m_{W_{n}} \sim m_{Z_{n}}$

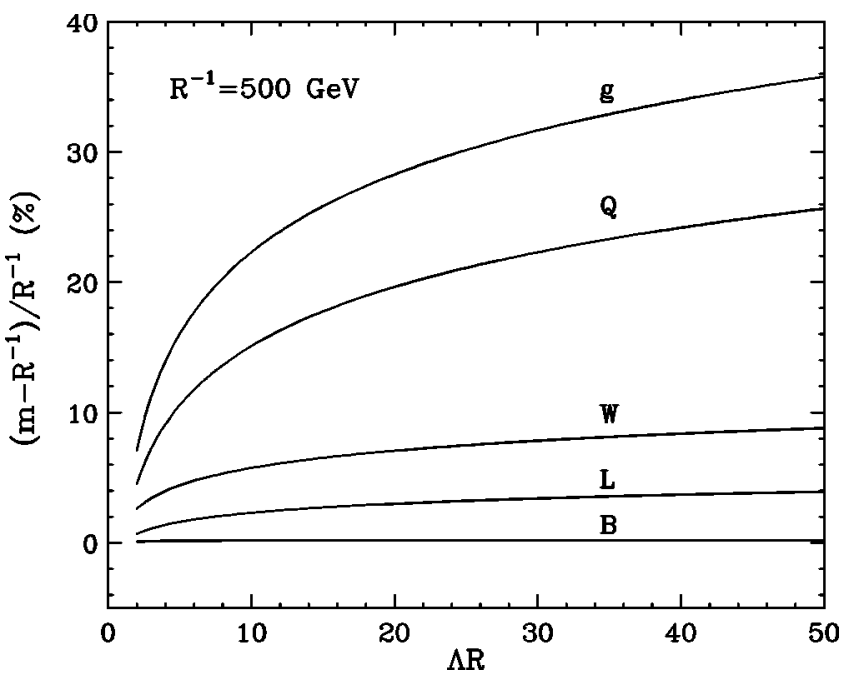

FIG. 2. Radiative corrections (in \%) to the spectrum of the first KK level for $R^{-1}=500 \mathrm{GeV}$, versus $\Lambda R$. 
$>m_{L_{n}}>m_{l_{n}}>m_{\gamma_{n}}$. The lightest KK particle $\gamma_{1}$, is a mixture of the first KK mode $B_{1}$ of the $U(1)_{Y}$ gauge boson $B$ and the first KK mode $W_{1}^{0}$ of the $S U(2)_{W} W^{3}$ gauge boson. (The possibility of the first level KK graviton being the LKP is irrelevant for collider phenomenology, since the decay lifetime of $\gamma_{1}$ to $G_{1}$ would be of cosmological scales.) We will usually denote this state by $\gamma_{1}$. However, note that the corresponding "Weinberg" angle $\theta_{1}$ is much smaller than the Weinberg angle $\theta_{W}$ of the standard model [10], so that the $\gamma_{1}$ LKP is mostly $B_{1}$ and $Z_{1}$ is mostly $W_{1}^{0}$. The mass splittings among the level $1 \mathrm{KK}$ modes are large enough for the prompt decay of a heavier level $1 \mathrm{KK}$ mode to a lighter level $1 \mathrm{KK}$ mode. But since the spectrum is still quite degenerate, the ordinary SM particles emitted from these decays will be soft, posing a challenge for collider searches.

The terms localized at the orbifold fixed points also violate the KK number by even units. However, assuming that no explicit KK-parity violating effects are put in by hand, KK parity remains an exact symmetry. The boundary terms allow higher $(n>1) \mathrm{KK}$ modes to decay to lower KK modes, and even level states can be singly produced (with smaller cross sections because the boundary couplings are volume suppressed). Thus $\mathrm{KK}$ number violating boundary terms are important for higher KK mode searches as we will discuss in Sec. IV.

\section{FIRST KK LEVEL}

Once the radiative corrections are included, the KK mass degeneracy at each level is lifted and the KK modes decay promptly. The collider phenomenology of the first KK level is therefore very similar to a supersymmetric scenario in which the superpartners are relatively close in mass-all squeezed within a mass window of $100-200 \mathrm{GeV}$ (depending on the exact value of $R$ ). Each level $1 \mathrm{KK}$ particle has an exact analogue in supersymmetry: $B_{1} \leftrightarrow b$-ino, $g_{1} \leftrightarrow$ gluino, $Q_{1}\left(q_{1}\right) \leftrightarrow$ left-handed (right-handed) squark, etc. The decay cascades of the level $1 \mathrm{KK}$ modes will terminate in the $\gamma_{1}$ LKP (Fig. 3). Just as the neutralino LSP is stable in $R$-parity conserving supersymmetry, the $\gamma_{1}$ LKP in MUEDs is stable due to KK parity conservation and its production at colliders results in generic missing energy signals.

It is known that supersymmetry with a stable neutralino LSP is difficult to discover at hadron colliders if the superpartner spectrum is degenerate. Hence the discovery of level $1 \mathrm{KK}$ modes in MUEDs at first sight appears problematic as well-the decay products resulting from transitions between level $1 \mathrm{KK}$ states may be too soft for reliable experimental observation at hadron colliders. This issue is the subject of this section.

Before we address the possible level 1 discovery channels in some detail, we need to determine the allowed decays at level 1 and estimate their branching fractions. For any given set of input parameters (3) the mass spectrum and couplings of the KK modes in MUEDs are exactly calculable [10]. Hence one obtains very robust predictions for the main branching ratios of interest for phenomenology.

KK gluon. The heaviest KK particle at level 1 is the KK gluon $g_{1}$. Its two-body decays to KK quarks $Q_{1}$ and $q_{1}$ are

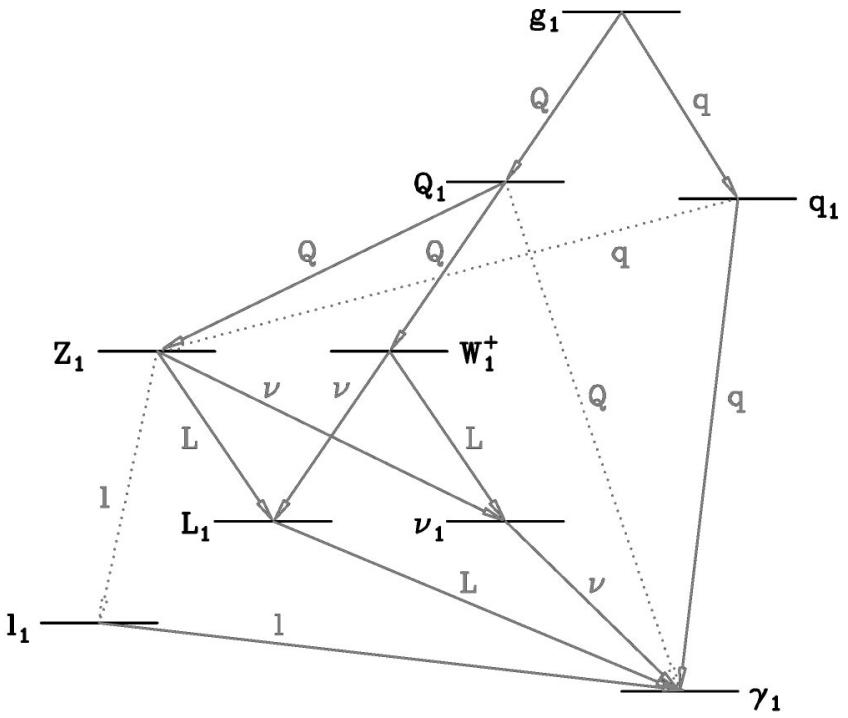

FIG. 3. Qualitative sketch of the level $1 \mathrm{KK}$ spectroscopy depicting the dominant (solid) and rare (dotted) transitions and the resulting decay product.

always open and have similar branching fractions: $B\left(g_{1}\right.$ $\left.\rightarrow Q_{1} Q_{0}\right) \simeq B\left(g_{1} \rightarrow q_{1} q_{0}\right) \simeq 0.5$.

$K K$ quarks. The case of $S U(2)$-singlet quarks $\left(q_{1}\right)$ is very simple - they can only decay to the hypercharge gauge boson $B_{1}$, hence their branchings to $Z_{1}$ are suppressed by the level 1 Weinberg angle $\theta_{1} \ll \theta_{W}: B\left(q_{1} \rightarrow Z_{1} q_{0}\right) \simeq \sin ^{2} \theta_{1} \sim 10^{-2}$ $-10^{-3}$ while $B\left(q_{1} \rightarrow \gamma_{1} q_{0}\right) \simeq \cos ^{2} \theta_{1} \sim 1$. Thus $q_{1}$ production yields jets plus missing energy, the exception being $t_{1}$ $\rightarrow W_{1}^{+} b_{0}$ and $t_{1} \rightarrow H_{1}^{+} b_{0}$ (the latter will be in fact the dominant source of $H_{1}^{+}$production at hadron colliders).

$S U(2)$-doublet quarks $\left(Q_{1}\right)$ can decay to $W_{1}^{ \pm}, Z_{1}$ or $\gamma_{1}$. In the limit $\sin \theta_{1} \ll 1, S U(2)_{W^{-}}$-symmetry implies

$$
B\left(Q_{1} \rightarrow W_{1}^{ \pm} Q_{0}^{\prime}\right) \simeq 2 B\left(Q_{1} \rightarrow Z_{1} Q_{0}\right)
$$

and furthermore for massless $Q_{0}$ we have

$$
\frac{B\left(Q_{1} \rightarrow Z_{1} Q_{0}\right)}{B\left(Q_{1} \rightarrow \gamma_{1} Q_{0}\right)} \simeq \frac{g_{2}^{2} T_{3 Q}^{2}\left(m_{Q_{1}}^{2}-m_{Z_{1}}^{2}\right)}{g_{1}^{2} Y_{Q}^{2}\left(m_{Q_{1}}^{2}-m_{\gamma_{1}}^{2}\right)}
$$

where $g_{2}\left(g_{1}\right)$ is the $S U(2)_{W}\left[U(1)_{Y}\right]$ gauge coupling, and $T_{3}$ and $Y$ stand for weak isospin and hypercharge, correspondingly. We see that the $Q_{1}$ decays to $S U(2)$ gauge bosons, although suppressed by phase space, are numerically enhanced by the ratio of the couplings and quantum numbers. With typical values for the mass corrections from Fig. 2, Eqs. (4) and (5) yield $B\left(Q_{1} \rightarrow W_{1}^{ \pm} Q_{0}^{\prime}\right) \sim 65 \%, B\left(Q_{1}\right.$ $\left.\rightarrow Z_{1} Q_{0}\right) \sim 33 \%$ and $B\left(Q_{1} \rightarrow \gamma_{1} Q_{0}\right) \sim 2 \%$.

$K K W$ - and $Z$-bosons. With their hadronic decays closed, $W_{1}^{ \pm}$and $Z_{1}$ decay democratically to all lepton flavors: $B\left(W_{1}^{ \pm} \rightarrow \nu_{1} L_{0}^{ \pm}\right)=B\left(W_{1}^{ \pm} \rightarrow L_{1}^{ \pm} \nu_{0}\right)=\frac{1}{6} \quad$ and $\quad B\left(Z_{1} \rightarrow \nu_{1} \bar{\nu}_{0}\right)$ $=B\left(Z_{1} \rightarrow L_{1}^{ \pm} L_{0}^{\mp}\right) \simeq \frac{1}{6}$ for each generation. $Z_{1} \rightarrow l_{1}^{ \pm} l_{0}^{\mp}$ decays are suppressed by $\sin ^{2} \theta_{1}$.

KK leptons. The level $1 \mathrm{KK}$ modes of the charged leptons as well as the neutrinos decay directly to $\gamma_{1}$. As a result $W_{1}^{ \pm}$ 
and $Z_{1}$ always effectively decay as $W_{1}^{ \pm} \rightarrow \gamma_{1} L_{0}^{ \pm} \nu_{0}$ and $Z_{1}$ $\rightarrow \gamma_{1} L_{0}^{ \pm} L_{0}^{\mp}$ or $Z_{1} \rightarrow \gamma_{1} \nu_{0} \bar{\nu}_{0}$, with relatively large $e$ and $\mu$ yields.

KK Higgs bosons. Their decays depend on their masses. They can decay into the KK $W, Z$ bosons or KK $t, b$ quarks if they are heavier and the phase space is open. On the other hand, if they are lighter than $W_{1}, Z_{1}, t_{1}, b_{1}$ (as in the example of Fig. 1), their tree-level two-body decays will be suppressed. Then they will decay to $\gamma_{1}$ and the corresponding virtual zero-level Higgs boson, or to $\gamma_{1} \gamma_{0}$ through a loop.

We are now able to discuss the optimum strategy for MUEDs KK searches at hadron colliders. Level $1 \mathrm{KK}$ states necessarily have to be pair produced, due to KK parity conservation. The approximate mass degeneracy at each level ensures that strong production dominates, with all three subprocesses (quark-quark, quark-gluon and gluon-gluon) having comparable rates $[8,12]$.

For an estimate of the reach at the Tevatron or the LHC, we need to discuss the final state signatures and the related backgrounds. The signature with the largest overall rate is $\boldsymbol{E}_{T}+N \geqslant 2$ jets, which is similar to the traditional squark and gluino searches [13]. It arises from inclusive (direct or indirect) $q_{1} q_{1}$ production. Roughly one quarter of the total strong production cross-section $\sigma_{\text {tot }}^{\text {had }}$ materializes in $q_{1} q_{1}$ events. However, in spite of the large missing mass in these events, the measured missing energy is rather small, since it is correlated with the energy of the relatively soft recoiling jets. As a conservative rough guide for the discovery reach we can use existing studies of the analogous supersymmetric case. One might expect that Run II can probe $R^{-1}$ $\sim 300 \mathrm{GeV}$ [14] while the LHC reach for $R^{-1}$ is no larger than $1.2 \mathrm{TeV}$ [15]. While the jetty signatures can be potentially used for discovery, further studies in an MUEDs context are needed. Here we prefer to discuss the much cleaner multilepton final states arising from diboson $\left(W_{1}^{ \pm}\right.$or $\left.Z_{1}\right)$ production.

Consider inclusive $Q_{1} Q_{1}$ production, whose cross-section also roughly equals $\frac{1}{4} \sigma_{\text {tot }}^{\text {had }}$. The subsequent decays of $Q_{1}$ 's yield $W_{1}^{ \pm} W_{1}^{ \pm}, W_{1}^{ \pm} Z_{1}$ and $Z_{1} Z_{1}$ pairs in proportion $4: 4: 1$. The $W_{1}^{ \pm}$and $Z_{1}$ decays in turn provide multilepton final states with up to 4 leptons plus missing energy, all of which may offer the possibility of a discovery. In the following we concentrate on the gold-plated $4 l \boldsymbol{E}_{T}$ signature.

We shall conservatively ignore additional signal contributions from direct diboson production and $Q_{1} W_{1}^{ \pm}$or $Q_{1} Z_{1}$ processes. For the Tevatron we use the single lepton triggers $p_{T}(l)>20 \mathrm{GeV}$ and $|\eta(e)|<2.0,|\eta(\mu)|<1.5$; or the missing energy trigger $\boldsymbol{E}_{T}>40 \mathrm{GeV}$. Because the channel is very clean, we use relatively soft off-line cuts, $p_{T}(l)$ $>\{15,10,10,5\} \mathrm{GeV},|\eta(l)|<2.5$ and $\mathbb{E}_{T}>30 \mathrm{GeV}$. The remaining physics background comes from $Z Z \rightarrow l^{ \pm} l^{\mp} \tau^{+} \tau^{-}$ $\rightarrow 4 l \boldsymbol{E}_{T}$ where $Z$ stands for a real or virtual $Z$ or $\gamma$ [16], and can be reduced by invariant mass cuts for any pair of opposite sign, same flavor leptons: $\left|m_{l l}-M_{Z}\right|>10 \mathrm{GeV}$ and $m_{l l}$ $>10 \mathrm{GeV}$. As a result, the expected background is less than 1 event in all of Run II and we require 5 signal events for discovery. The reach is shown in Fig. 4. We see that Run IIb

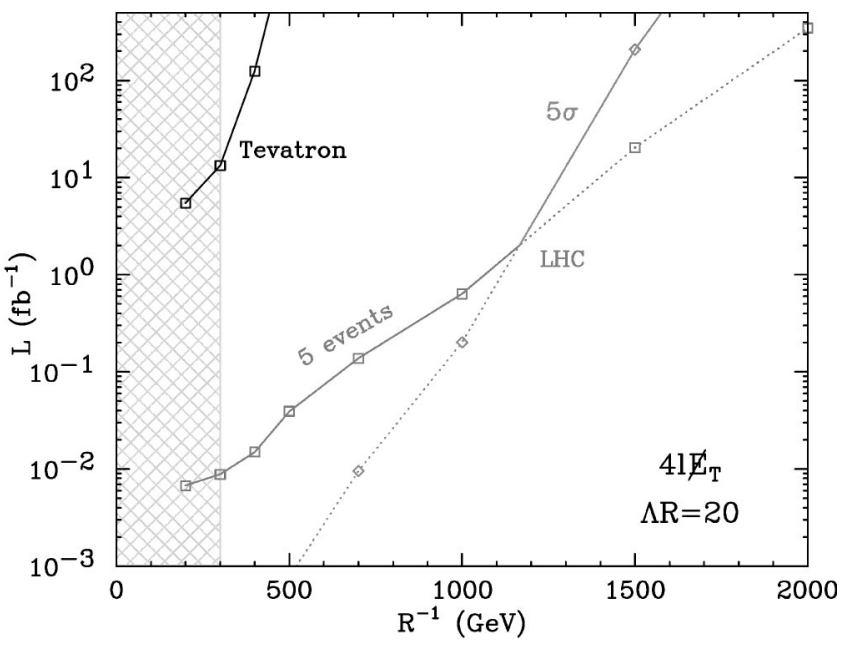

FIG. 4. Discovery reach for MUEDs at the Tevatron and the LHC in the $4 l \boldsymbol{E}_{T}$ channel. We require a $5 \sigma$ excess or the observation of 5 signal events, and show the required total integrated luminosity per experiment (in $\mathrm{fb}^{-1}$ ) as a function of $R^{-1}$, for $\Lambda R$ $=20$. (In either case we do not combine the two experiments.)

of the Tevatron will go slightly beyond the current indirect bounds $\left(R^{-1}>300 \mathrm{GeV}\right)$ from precision data [1].

For the LHC we use $p_{T}(l)>\{35,20,15,10\} \mathrm{GeV}$ with $|\eta(l)|<2.5$, which is enough for the single lepton trigger. In addition, we require $\boldsymbol{E}_{T}>50 \mathrm{GeV}$ and the same dilepton invariant mass cut. There are now several relevant background sources, including multiple gauge boson and/or top quark production [17], fakes, leptons from $b$-jets etc. We conservatively assume a background level of 50 events after cuts per $100 \mathrm{fb}^{-1}$ (1 year of running at high luminosity). Our LHC reach estimate is presented in Fig. 4. Without combining experiments, we plot the total integrated luminosity $L$ required for either an observation of 5 signal events or a $5 \sigma$ excess over the background. The reach, shown as a solid line, is defined as the larger of the two and extends to $R^{-1}$ $\sim 1.5 \mathrm{TeV}$.

Other leptonic channels such as two or three leptons with $\mathbb{E}_{T}$ may also be considered. They have more backgrounds but take advantage of the larger branching fraction for $Q_{1}$ $\rightarrow W_{1}^{ \pm} Q_{0}^{\prime}$ and offer higher statistics, which may prove useful especially for the case of the Tevatron.

In conclusion, note that at a hadron collider all signals from level $1 \mathrm{KK}$ states look very much like supersymmetry-all SM particles have "partners" with similar couplings, and identifying the extra-dimensional nature of the new physics becomes rather challenging. Nevertheless, there are three features which distinguish the MUEDs scenario from ordinary supersymmetry. First, the spins are different, but this "bosonic" nature of the newly discovered "supersymmetry" will most likely escape detection at a hadron collider. Second, the analogy with the MSSM is incomplete, as MUEDs do not have analogues of the "heavy" Higgs bosons of the MSSM. To be more precise, the level 1 KK modes of the Higgs bosons have exactly the same gauge quantum numbers as the MSSM Higgs bosons $H^{0}, A^{0}, H^{ \pm}$. But since they carry KK parity, their behavior is similar to that of Higgsinos instead. Now recall that there are regions 
of the MSSM parameter space where the LHC can only discover the SM-like Higgs boson, and misses the other three Higgs states of the MSSM. MUEDs could easily be confused with this scenario. This leaves us with the single smoking gun signature for MUEDs - the presence of higher level KK modes.

\section{SECOND KK LEVEL}

Through KK number preserving interactions a level $2 \mathrm{KK}$ state can decay to two level $1 \mathrm{KK}$ modes, or to another level 2 KK state and a SM particle. For example, the level 2 fermion decay widths (for massless $f_{0}$ and in leading order of $\hat{\delta} m$ ) are easily computed at tree level using the Feynman rules for KK modes. We find

$\Gamma\left(f_{2} \rightarrow V_{2} f_{0}\right) \approx \frac{3 c^{2} g^{2} m_{f_{2}}}{8 \pi}\left(\frac{\hat{\delta} m_{f_{2}}}{m_{2}}-\frac{\hat{\delta} m_{V_{2}}}{m_{2}}\right)^{2}$,

$\Gamma\left(f_{2} \rightarrow V_{1} f_{1}\right) \approx \frac{11 c^{2} g^{2} m_{f_{2}}}{16 \sqrt{2} \pi}\left(\frac{\hat{\delta} m_{f_{2}}}{m_{2}}-\frac{\hat{\delta} m_{V_{1}}}{2 m_{1}}-\frac{\hat{\delta} m_{f_{1}}}{2 m_{1}}\right)^{3 / 2}$,

where $\hat{\delta} m$ represents the total mass correction and $c$ is a Clebsch factor. For a level 2 gauge boson

$\Gamma\left(V_{2} \rightarrow f_{2} f_{0}\right) \approx \frac{c^{2} g^{2} m_{V_{2}}}{4 \pi}\left(\frac{\hat{\delta} m_{V_{2}}}{m_{2}}-\frac{\hat{\delta} m_{f_{2}}}{m_{2}}\right)^{2}$

$\Gamma\left(V_{2} \rightarrow f_{1} f_{1}^{\prime}\right) \approx \frac{c^{2} g^{2} m_{V_{2}}}{6 \sqrt{2} \pi}\left(\frac{\hat{\delta} m_{V_{2}}}{m_{2}}-\frac{\hat{\delta} m_{f_{1}}}{2 m_{1}}-\frac{\hat{\delta} m_{f_{1}^{\prime}}}{2 m_{1}}\right)^{3 / 2}$,

counting both KK chiralities in the last case. All of the decays (6)-(9) are phase space suppressed, once again leaving rather little visible energy deposited in the detector.

A level 2 KK gauge boson, however, can also decay directly to two SM particles via KK number violating interactions [10]. The width is

$$
\Gamma\left(V_{2} \rightarrow f_{0} f_{0}\right) \approx \frac{c^{2} g^{2} m_{V_{2}}}{12 \pi}\left(\frac{\bar{\delta} m_{V_{2}}}{m_{2}}-\frac{\bar{\delta} m_{f_{2}}}{m_{2}}\right)^{2},
$$

where $\bar{\delta} m$ only contains the mass corrections due to the boundary terms (though typically $\hat{\delta} m \simeq \bar{\delta} m$ ). These decays are not phase space suppressed, and deposit a lot of energy, hence they offer the best opportunity for a level 2 discovery.

Level $2 \mathrm{KK}$ gauge bosons can be pair-produced through KK number preserving interactions, or singly produced through their suppressed KK number violating couplings to SM quarks and leptons. We first concentrate on the $g_{2}$ signal. Using Eqs. (8) $-(10)$, we find $B\left(g_{2} \rightarrow Q_{0} Q_{0}, q_{0} q_{0}\right) \simeq 0.1$. The production of $g_{2}$ in association with another level 2 colored particle then yields a unique $\boldsymbol{E}_{T}+N>2$ jet signature, where the invariant mass of the two leading jets reconstructs to $m_{g_{2}}$. In the absence of a problematic physics background, we require 10 events before cuts and efficiencies for discovery, leaving us with a reach for $R^{-1}$ of just below $1 \mathrm{TeV} . W_{2}^{ \pm}, Z_{2}$ and $\gamma_{2}$ searches in their hadronic modes will be very similar. Branching fractions to leptonic decay modes are very small and do not permit a significant reach. Notice that $\gamma_{2}$ has no KK preserving decay modes left open, hence $\Sigma_{f} B\left(\gamma_{2}\right.$ $\left.\rightarrow f_{0} f_{0}\right) \simeq 1$.

The usual $W^{\prime} / Z^{\prime}$ and coloron searches are sensitive to singly produced level $2 \mathrm{KK}$ gauge bosons. However, the reach is inferior due to the smallness of the KK number violating couplings, which are only a fraction of the SM gauge couplings-typically $10-20 \%$ for quarks and only a few percent for leptons.

\section{CONCLUSIONS}

Universal extra dimensions with compactification radius near the $\mathrm{TeV}$ scale promise exciting phenomenology for future colliders. All standard model particles have KK partners which can be produced with enormous cross sections at the LHC. As we showed in this paper, the detection of KK particles at the LHC is nontrivial as they decay to very soft standard model particles which are difficult (but not impossible) to see above background. Clearly, more realistic simulations of the phenomenology of MUEDs are necessary and studies for different values of the boundary couplings would be of interest as well.

A lepton collider running at the center of mass energy of the second level photon or $Z$ is ideal for measuring the small mass splittings between states and determining spins. However, the required center of mass energy $\left(\sim 2 R^{-1}\right)$ may be too high for the next generation linear collider.

Finally, we note that similarly to the neutralino LSP in supersymmetry, the $\gamma_{1}$ LKP of MUEDs is a great cold dark matter candidate, whose annihilation rate is not helicity suppressed. A study of the resulting abundance and detection opportunities is underway [18].

\section{ACKNOWLEDGMENTS}

We would like to thank M. Chertok, B. Dobrescu and B. Schumm for useful discussions, and D. E. Kaplan for suggesting the catchy title. We also thank the Aspen Center for Physics for hospitality during the initial stage of this work. H.-C.C. is supported by the Department of Energy grant DEFG02-90ER-40560. M.S. is supported in part by the Department of Energy under grant number DE-FG02-91ER-40676.
[1] T. Appelquist, H.-C. Cheng, and B.A. Dobrescu, Phys. Rev. D 64, 035002 (2001).

[2] N. Arkani-Hamed, H.-C. Cheng, B.A. Dobrescu, and L.J. Hall, Phys. Rev. D 62, 096006 (2000).
[3] T. Appelquist, B.A. Dobrescu, E. Ponton, and H.U. Yee, Phys. Rev. Lett. 87, 181802 (2001).

[4] B.A. Dobrescu and E. Poppitz, Phys. Rev. Lett. 87, 031801 (2001). 
[5] T. Appelquist, B.A. Dobrescu, E. Ponton, and H.U. Yee, Phys. Rev. D 65, 105019 (2002).

[6] K. Agashe, N.G. Deshpande, and G.H. Wu, Phys. Lett. B 514, 309 (2001).

[7] T. Appelquist and B.A. Dobrescu, Phys. Lett. B 516, 85 (2001).

[8] C. Macesanu, C. D. McMullen, and S. Nandi, Phys. Rev. D 66, 015009 (2002).

[9] D.A. Dicus, C.D. McMullen, and S. Nandi, Phys. Rev. D 65, 076007 (2002).

[10] H.-C. Cheng, K. T. Matchev, and M. Schmaltz, Phys. Rev. D 66, 036005 (2002).

[11] H. Georgi, A.K. Grant, and G. Hailu, Phys. Lett. B 506, 207
(2001).

[12] T.G. Rizzo, Phys. Rev. D 64, 095010 (2001).

[13] D0 Collaboration, B. Abbott et al., Phys. Rev. Lett. 83, 4937 (1999); CDF Collaboration, T. Affolder et al., ibid. 88, 041801 (2002).

[14] SUGRA Working Group Collaboration, S. Abel et al., hep-ph/0003154.

[15] S.I. Bityukov and N.V. Krasnikov, Phys. Lett. B 469, 149 (1999).

[16] K.T. Matchev and D.M. Pierce, Phys. Lett. B 467, 225 (1999).

[17] V.D. Barger, A.L. Stange, and R.J. Phillips, Phys. Rev. D 45, 1484 (1992).

[18] H.-C. Cheng, J.L. Feng, and K.T. Matchev, hep-ph/0207125. 